\title{
An Efficient Procedure to Identify and Quantify New Molecules for Insulating Gas Mixtures
}

\section{Journal Article}

Author(s):

Franck, Christian (D); Dahl, Dominik A.; Rabie, M.; Haefliger, P.; Koch, M.

Publication date:

2014

Permanent link:

https://doi.org/10.3929/ethz-a-010881816

Rights / license:

In Copyright - Non-Commercial Use Permitted

Originally published in:

Contributions to plasma physics 54(1), https://doi.org/10.1002/ctpp.201300030 
This is the pre-peer reviewed version of the following article:

C. M. Franck, D.A. Dahl, M. Rabie, P. Haefliger, and M. Koch, An Efficient Procedure to Identify and Quantify New Molecules for Insulating Gas Mixtures, Contrib. Plasma Phys., 1 - 11 (2013) / DOI 10.1002/ctpp.201300030,

which has been published in final form at

[http://onlinelibrary.wiley.com/doi/10.1002/ctpp.201300030/abstract].

This article may be used for non-commercial purposes in accordance with Wiley Terms and Conditions for Self-Archiving. 


\title{
An efficient procedure to identify and quantify new molecules for insulating gas mixtures
}

\author{
C. M. Franck ${ }^{* 1}$, D. A. Dahl ${ }^{1}$, M. Rabie ${ }^{1}$, P. Haefliger ${ }^{1}$, and M. Koch ${ }^{1}$ \\ ${ }^{1}$ Institute for Power Systems and High Voltage Technology, ETH Zurich, Physikstr. 3, CH-8092 Zurich
}

Received 1 May 2013, revised XX 2013, accepted XX 2013

Published online XX 2013

Key words High Voltage Insulation, SF6 replacements, Pulsed Townsend discharge, swarm parameters

\begin{abstract}
In this contribution, a new procedure to systematically identify and quantify novel molecular gases with low global warming potential for application in high voltage insulation as gas mixtures is presented. The attention is focused on highly efficient procedures to be able to scan a large number of candidate gases. To identify new molecules, we derived an empirical correlation between the electric strength of a gas and certain molecular properties, like polarizability or dipole moment, which can be calculated by means of density functional theory. The swarm parameters of these pre-selected molecules in mixtures with buffer gases is then quantified, using a newly set-up Pulsed Townsend experiment. The setup operates with a high degree of automation to enable systematic evaluation of gas mixtures not to miss possible synergistic effects. Key element of this PT setup is a new photocathode that works with a high quantum efficiency and long lifetime even when exposed to reactive species during the measurements. Moreover, for an automated operation it is important to know precisely in which range the experiment can be operated, i.e. for example to know up to which electron density space charge effects can be neglected. Finally, the measured swarm parameters need to be translated into breakdown voltage strengths of different electrode arrangements and different applied voltage wave shapes. For this, a model of the the streamer to leader transition in SF6 will be applied to general strong electronegative gases in future studies.
\end{abstract}

Copyright line will be provided by the publisher

\section{Introduction}

Today, predominantly sulfur hexafluoride $\left(\mathrm{SF}_{6}\right)$ is used in high-voltage gas insulated equipment (GIS). It combines a uniquely high electric strength with long term stability and easy handling. However, since it has become clear that $\mathrm{SF}_{6}$ is one of the most potent greenhouse gases (GWP 23'900), several attempts have been made to find alternative insulation gases and gas mixtures. To date, only synthetic air [1] and $\mathrm{SF}_{6} / \mathrm{N}_{2}$ [2] mixtures are seriously accepted alternatives. Besides that, numerous other gases have been investigated and partly compared $[3,4,5,6]$. However, a systematic search strategy to find alternatives has not been developed.

Most of the replacement candidate gases are liquid at typical operating pressures of GIS $(0.5 \ldots 0.7 \mathrm{MPa})$ and ambient temperatures of $-20^{\circ} \mathrm{C}$. They can thus only be used with reduced partial pressures and a second (buffer) gas must be added. Of these buffer gases, $\mathrm{N}_{2}, \mathrm{CO}_{2}, \mathrm{O}_{2}$ and some noble gases are the most favourable ones. The buffer gas is not only useful to increase the filling and operating pressure. Synergistic effects between the electronegative and the buffer gas can lead to a higher electric strength compared to the electronegative gas alone at the corresponding partial pressure [29,30]. The most interesting synergy effect is due to the influence of the buffer gas on the electron energy distribution function (eedf). The resulting attachment rates are large if the eedf of the gas mixture has significant components in the energy range where attachment cross sections are large.

Consequently, a vast number of potential replacement mixtures has to be analyzed: a large number of different strong electron attaching candidate gases in mixture with a few selected buffer gases at varying mixing ratios. This fact enforces the need for highly efficient identification and quantification methods.

In this contribution, we report on an efficient procedure to identify and quantify alternative gas mixtures that could possibly replace $\mathrm{SF}_{6}$ in high voltage GIS. It basically consists of three parts: i) a systematic and efficient pre-screening method to identify a small number of molecules (as the electron attaching gas within mixtures) with high electric strength, low boiling point and low global warming potential, ii) an efficient method to quantify the

* Corresponding author: e-mail: cfranck@ethz.ch 
interaction of these molecules with buffer gases, and iii) to quantify the electric strength (or breakdown voltage) of the gas mixture for all technical relevant geometrical conditions and voltage wave shapes.

The pre-screening method is based on empirically found predictor functions that are constructed from molecular properties (like polarizability, dipole moment,...) which can be computationally calculated by means of quantum-chemical methods [7].

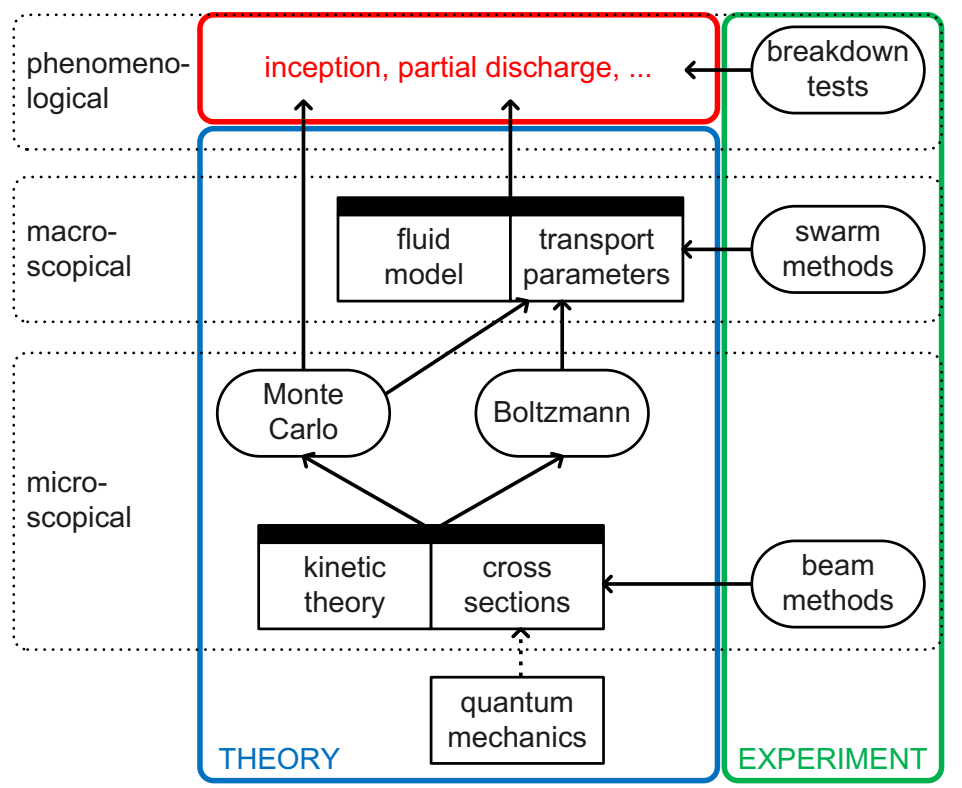

Fig. 1 Overview of the principal different approaches to systematically investigate different insulation gases.

The methods to quantify the electric strength can be grouped in three basic categories: phenomenological, macroscopic, and microscopic ones, like shown in Fig. 1. Direct breakdown experiments yield the electric strength of a gas or gas mixture $[8,9,10,11]$. A certain electrode arrangement, typically needle-plane, coaxial or parallel planes, is chosen, and the breakdown voltage level is measured for a particular wave shape. A large number of breakdown experiments has to be carried out to properly account for the statistical nature of the breakdown process and it has to be ensured that each measurement is independent of the previous ones $[12,13,14]$. The resulting information is only valid for this configuration and wave shape and cannot be generalized or otherwise transferred.

The other extreme would be to apply microscopic methods with the maximum of information possible, i.e. the complete set of cross sections for the interaction of electrons with the gas particles have to be known. Such cross sections can be measured using beam methods $[15,16]$. In particular cases, the cross sections can be determined $\mathrm{ab}$ intio [17]. However, it turns out that beam methods and quantum mechanics are generally not sufficient to produce a set of cross sections consistent with the phenomena [18]. As the production of cross sections is a very resource-intensive and time-consuming work, one cannot expect to quickly come to conclusions on novel gases using microscopical methods.

For the macroscopic methods, the input consists of a set of transport parameters [13]. For the scope of discharges in technical equipment, it is usually sufficient to know the parameters of electron transport, energy transport, momentum transport, and the transport parameters for ions and excited gas species. We have decided to use an experimental swarm method, the pulsed Townsend (PT) method, to provide measurements of the electron and ion transport parameters [19]. The PT method is a traditional experiment for measuring electron swarm parameters $[20,21]$. Its main advantage is that it permits direct observation of the spatiotemporal evolution of an electron swarm yielding at the same time multi-parameter sets of swarm coefficients. Special focus in our design was put on a high degree of automation so that large sets of gases and gas mixtures can be measured efficiently. Key element was a novel electron source with a high quantum efficiency so that reliable, low-energy, pulsed UV-laser systems can be utilized. 
The resulting macroscopic swarm parameters of gas mixtures need to be related to breakdown voltages in GIS. For this, breakdown models have to be derived and validated. Ideally, these models are valid for all strongly electronegative gases and use the measured swarm parameters as input.

The three steps of our procedure are presented in detail in the following sections: The efficient pre-selection procedure is briefly outlined in section 2. The automated PT setup is introduced in section 3: new results on the specially developed photocathode are presented in section 3.2, example measurement results of the effective ionization rates of $\mathrm{C}_{3} \mathrm{~F}_{8}$ and $\mathrm{SF}_{6}$ in $\mathrm{Ar}$ and $\mathrm{N}_{2}$ in section 3.3, and new measurements on the role of charge density show the operation regimes in section 3.4. Finally, a breakdown model that enables the prediction of the breakdown voltages in GIS for all relevant configurations and wave shapes using the measured swarm parameters is outlined in section 4. A short summary concludes this paper in section 5.

\section{Efficient Pre-Selection}

A mandatory prerequisite for an efficient procedure is an effective identification method to filter out a few most promising candidate molecules for subsequent experimental identification. In other fields of research, as e.g. in medical sciences or economic statistics, it is very common to identify the relationship between descriptor variables (independent variables) with phenomenological quantities (dependent variables) by statistical methods $[22,23]$. The descriptor variables can either be determined experimentally or, preferably, calculated with numerical methods. In a previous report [7] a method was presented that uses such numerically calculated predictor variables to derive an estimation on the relative electric strength $(\mathrm{ES})$ compared to $\mathrm{SF}_{6}\left(E_{r}\right)$ and the boiling point $\left(T_{B}\right)$. The descriptor values were molecular properties derived from density functional theory (DFT) calculations.

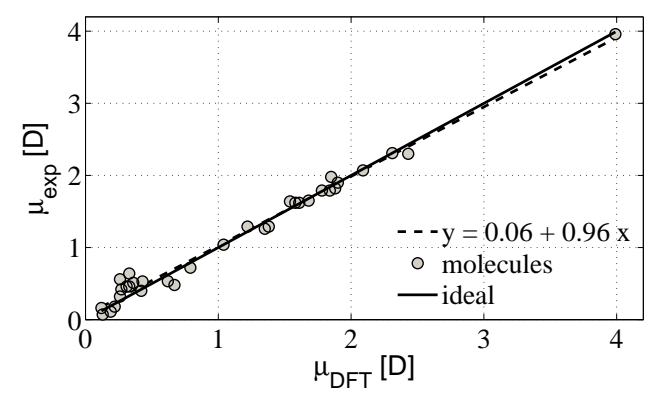

Fig. 2 Measured vs. calculated electric dipole moment $\mu$ for 33 molecules. The dashed line is the regression between measured and calculated values and results in $\mu_{\exp }=0.06+0.96 \mu_{\text {calc }}$. The solid line corresponds to the perfect agreement $\mu_{\exp }=\mu_{\mathrm{DFT}}$

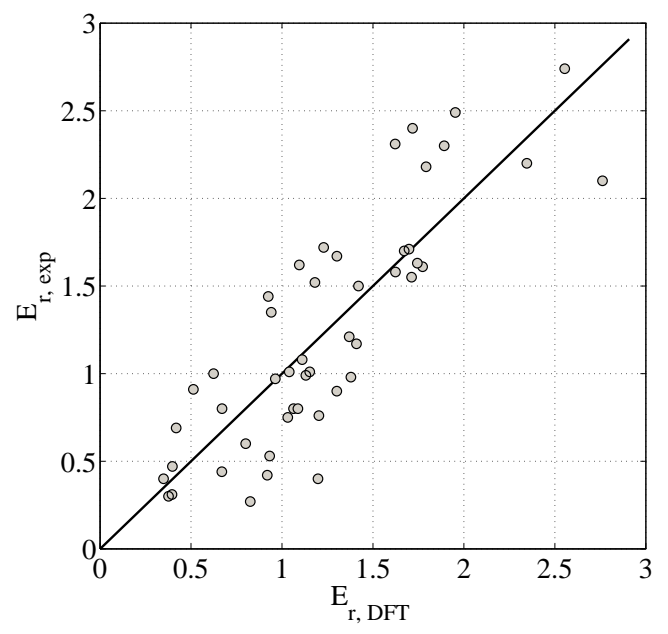

Fig. 3 Measured vs. predicted relative electric strength $E_{r}$ for 48 polar molecules. The solid line corresponds to the ideal correlation $E_{\mathrm{r} \text {,meas }}=E_{\mathrm{r}, \mathrm{DFT}}$.

From literature $[25,4,26,27]$ a set of 48 polar and 19 non-polar molecules has been extracted for which the electric strength is known. The descriptor variables found to be important are: a) average static electronic polarizability $\alpha$, b) electric dipole moment $\mu, \mathrm{c}$ ) vertical ionization energy $\epsilon_{i}^{v}$, d) adiabatic ionization energy $\epsilon_{i}^{a}$, e) vertical electron affinity $\epsilon_{a}^{v}$, f) adiabatic electron affinity $\epsilon_{a}^{a}$, and g) electron number $N_{e}$. The molecular structure is optimized in the electronic ground state neglecting vibrational and temperature corrections. The calculations are performed with the Turbomole program [24]. For the majority of our set of 67 molecules, experimental results for the above listed molecular properties can be found in literature. It is thus possible to analyze the correlation between measured and calculated properties. Fig. 2 shows such a correlation on the example of the dipole moments. The linear regression is $\mu_{\exp }=0.06+0.96 \mu_{\text {calc }}$ with a standard deviation of 
$\sigma=0.11 \mathrm{D}$ (Debye). Similarly good correlations (though partly with a larger constant offset) was found for the other molecular properties [7]. It is thus meaningful to use the numerically calculated descriptor variables.

This set served as the basis for a regression analysis that identifies the strongest correlation between the known relative electric strength and an empirical function based on the calculated descriptor variables. The resulting expressions are found purely empirical and are given (for polar molecules) by:

$$
\begin{aligned}
& E_{r}=-1.05+3.8 \cdot 10^{-3} \mu^{0.3} N_{e}^{1.3}+5.6 \cdot 10^{-4} \alpha^{0.6} \epsilon_{i}^{a 2.8} \\
& T_{B}=3370+270 \alpha^{0.23} \mu^{0.04}-2830 \epsilon_{i}^{a 0.07} m^{0.01}
\end{aligned}
$$

Fig. 3 shows this correlation on the example of the relative electric strength for polar molecules. The confidence of this regression is $R^{2}=0.71$ with a standard deviation of $\sigma=0.35$ with respect to the measured values. The regression analysis for non-polar molecules was even better with an $R^{2}=0.92$ and a standard deviation of $\sigma=0.18$ on the selected subset of 19 molecules.

Large sets of possible candidate molecules can now be pre-screened, preferably those with potentially large electron attaching cross sections. Even random or systematic combinatoric searches can be performed to find gases with high electric strength and low boiling point. The most suitable candidates have to be analyzed further to estimate their suitability with respect to other required characteristics like global warming potential, toxicity and potentially harmful dissociation products. An analysis similar to the one presented above for the electric strength and boiling point was made for the global warming potential for a number of stable, non-toxic and non-flammable new refrigerant fluids [28].

\section{Efficient Swarm-Parameter Measurement}

The subset of candidate molecules which is identified (e.g. by the pre-screening method from section 2) must be experimentally investigated to make a first assessment on their relative electric strength. One of the most meaningful parameters to compare the pure gas breakdown in homogeneous electric fields is the effective ionization coefficient $\alpha^{\star}=\alpha-\eta$ and, derived from this, the critical electric field strength $E_{\text {crit. }}$. In this step, also synergy effects shall be quantified, i.e. of a large number of candidate gases with several background gases in a wide range of mixing ratios must be investigated. For an efficient characterization, a PT setup with dedicated control software for running measurements in automated processes has been established [19]. In the following chapter, the newly build, highly automated, PT experiment is described, the new photocathode as the key component is characterized in detail, the exact operating regime is identified and example measurements are presented.

\subsection{Automated Pulsed Townsend Experiment}

The PT setup (cf. Fig. 4) consists basically of two parallel-plane electrodes at spacing $d$ inside a cylindrical vacuum chamber which is filled with a gas sample. The vacuum chamber is made from stainless steel and has a base pressure $<10^{-5} \mathrm{~Pa}$. The profile of the two polished stainless steel electrodes with radius $r_{0}=56 \mathrm{~mm}$ is of

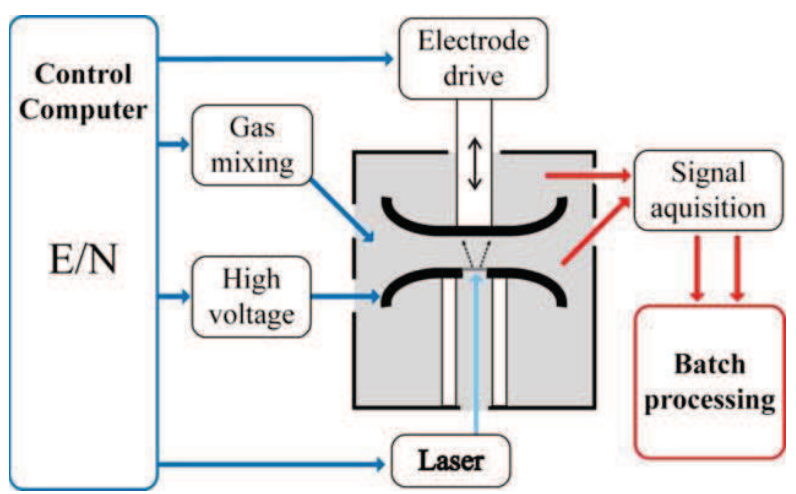

Fig. 4 Schematic representation of the automated PT setup. 
Rogowski type and a cylindrical symmetric electric field is produced between them which is almost homogeneous for $r<20 \mathrm{~mm}$ at spacings $d \leq 18 \mathrm{~mm}$. The electrode spacing $d$ can be varied by actuation of a motor driven micrometer screw at the anode in steps of $6 \mu \mathrm{m}$. By a short laser pulse an ensemble of electrons is generated at the cathode. It drifts through the sample gas at constant average velocity in a homogeneous electric field $E_{\mathrm{z}}$. The collective motion of these electrons under the influence of their interactions with the gas molecules gives rise to a displacement current which is recorded and analyzed. In contrast to previous experiments, a highly efficient photocathode is used to release a large number of initial electrons. By this, the needed laser pulse energy could be considerably reduced which enables to use a diode pumped laser system with long-term stability and large number of pulse operations. This photocathode, the key element to enable the high degree of automation of a PT experiment, is introduced and characterized in the next section.

\subsection{Improved Photocathode}

In the present context, a pulsed electron source (PES) consists of a photocathode and a pulsed laser system. Usually, photocathodes are operated in vacuum [33, 34, 35]. However, in PT experiments [36, 37, 38, 39], the photocathode necessarily operates in the presence of gases at $100 \mathrm{~Pa}$ to $100 \mathrm{kPa}$ total pressure. Under these conditions, one must consider the adsorption of gas molecules on the photocathode surface [40], and the interaction of photoelectrons with the gas particles in the immediate vicinity of the cathode. A photocathode for PT applications is required to meet the following conditions: 1) it should withstand the ions and radicals, which are generated from the sample gases in the PT discharge, 2) it must not disturb the homogeneous electric field within the PT setup, 3) it should be conductive with a low-ohmic, high-bandwidth electrical bonding, and 4) a large active area of the photocathode is desired.

A well established PES for PT applications uses a bulk metal photocathode. In many cases a stainless steel cathode was used in conjunction with a $266 \mathrm{~nm}$ laser system [41, 36, 37]. In other cases an aluminum cathode was used with a $355 \mathrm{~nm}$ laser [38] or a $337 \mathrm{~nm}$ laser [39]. In these cases the laser pulse energy used to be between $120 \mu \mathrm{J}$ and $20 \mathrm{~mJ}$.

For an efficient and automated measurement procedure, the pulsed laser system must operate reliably and stable for a large number of pulses. This is typically the case for low energy $(<200 \mu \mathrm{J})$ laser systems. Thus, we are particularly interested in thin film photocathodes. They generally have a lower electric work function $\Phi$ than the corresponding bulk material [33], which increases the quantum efficiency QE (ratio of released electrons to number of incident photons) at the laser wavelength.

There are previous reports on successful implementations of a PES in PT experiments with a thin film photocathode in a transmissive configuration: Gold (Au) [42, 43], Aluminum (Al) [44], and Palladium (Pd) [45].

The QE of a PES can be strongly influenced by the interaction between gas species and the cathode surface. In the case of an $\mathrm{Al}$ cathode, oxygen adsorption can cause a decrease of $\Phi$, and consequently, $\Phi$ increases when the adsorbed oxygen is removed due to the PT discharge [44]. In the case of a Au cathode, the QE of the PES collapses in a discharge containing nitrogen.

In this contribution we report on measurements with several different photocathodes that have been produced with a photo-active film of gold $(\mathrm{Au})$ and palladium $(\mathrm{Pd})$. The film thickness was varied in a range between 10 and $15 \mathrm{~nm}$. The samples were investigated for their transmission spectrum, their photocurrent spectrum, and their QE.

Table 1 Characteristic photon absorption length $\sigma$ of thin films for $\lambda=245 \mathrm{~nm}$ and $\lambda=437 \mathrm{~nm}$.

\begin{tabular}{lcc}
\hline material & $\sigma_{245}$ & $\sigma_{437}$ \\
\hline $\mathrm{Au}$ & $8.6-8.9 \mathrm{~nm}$ & $11.7-12.1 \mathrm{~nm}$ \\
$\mathrm{Pd}$ & $8.3-8.6 \mathrm{~nm}$ & $7.3-7.5 \mathrm{~nm}$ \\
\hline
\end{tabular}

The transmission as a function of the film thickness was evaluated for the mean absorption path $\sigma$ using the Beer-Lambert law. The $\sigma$-results of $\mathrm{Au}$ and $\mathrm{Pd}$ are in the order of $7 \ldots 12 \mathrm{~nm}$ and are listed in Table 1. A reasonable film thickness is thus $10 \ldots 20 \mathrm{~nm}$. A lower film thickness results in a lower electron number density as less radiation is absorbed. Increasing the film thickness results in a lower electron number density as some of the released electrons are re-absorbed inside the metal film and do not reach the surface. 
Table 2 Measurement results of the electron work function $\Phi$ for selected film materials (film thickness is given in brackets). $\mathrm{QE}_{0}$ is the photocathode efficiency at $245 \mathrm{~nm}$ in vacuum, and $\mathrm{QE}_{\text {eff }}$ is measured in gases at $266 \mathrm{~nm}$ for typical experimental conditions ( $p=20 \mathrm{mbar}, E / N=30 \mathrm{Td})$.

\begin{tabular}{lllll}
\hline film & & $\mathrm{QE}_{0}$ & \multicolumn{2}{c}{$\mathrm{QE}_{\text {eff }} \times 10^{-7}$} \\
\cline { 3 - 5 }$(\mathrm{nm})$ & $\Phi / \mathrm{eV}$ & $\times 10^{-5}$ & in $\mathrm{N}_{2}$ & in $\mathrm{CO}_{2}$ \\
\hline $\mathrm{Au}(13)$ & $4.80 \pm .04$ & 1.7 & 0.29 & 2.4 \\
$\mathrm{Pd}(10)$ & $4.77 \pm .05$ & 1.3 & 2.0 & 1.0 \\
$\mathrm{Pd}(13)$ & $4.74 \pm .04$ & 2.4 & & 1.1 \\
$\mathrm{Pd}(15)$ & $4.70 \pm .05$ & 0.89 & 3.9 & 2.5 \\
\hline
\end{tabular}

The photocurrent spectra $I_{\mathrm{ph}}(\lambda)$ has been recorded as a function of wavelength $\lambda$. The shape of $I_{\mathrm{ph}}(\lambda)$ was analyzed, using the expansion of the Fowler function (eqs. (6) and (7) from [46]). The resulting work functions $\Phi$ are listed in the second column of Table 2. Photocurrent measurements have been made using light sources with well determined spectral intensity. The quantum efficiencies have been derived in vacuum $\left(\mathrm{QE}_{0}\right)$ and with different gases in the PT setup $\left(\mathrm{QE}_{\text {eff }}\right)$. These are listed in the last three columns of Table 2.

Our results indicate that $\mathrm{Au}$ and Pd films have very similar optical properties in the spectral range $250 \mathrm{~nm}$ $\leq \lambda \leq 300 \mathrm{~nm}$, where the two film materials approximately agree in the transmissivity, $\Phi$ and $\mathrm{QE}_{0}$.

An important difference between $\mathrm{Au}$ and $\mathrm{Pd}$ photocathodes appeared with operation in the presence of $\mathrm{N}_{2}$, where the $\mathrm{QE}_{\text {eff }}$ of $\mathrm{Au}$ photocathodes was found to collapse (see Table 2). Probably, the decrease of the $\mathrm{QE}_{\mathrm{eff}}$ is caused by reactions on the Au surface between adsorbed species and $\mathrm{N}_{2}^{+}$or $\mathrm{N}_{2}$ metastables.

Another important advantage of Pd- over Au-films was observed with respect to the regeneration of $\mathrm{QE}_{\mathrm{eff}}$ after contamination. After the active surface of the $\mathrm{Pd}$ cathode was flushed with $\mathrm{H}_{2}$, the $\mathrm{QE}_{\text {eff }}$ increases dramatically. A reduced $\mathrm{QE}_{\text {eff }}$ due to contaminating gases was regenerated within minutes. After hydrogenization, the vacuum pressure in the PT setup increased by about $4 \cdot 10^{-6} \mathrm{~Pa}$. Outgassing of $\mathrm{H}_{2}$ thus only weakly pollutes the gas sample.

\subsection{Swarm parameter measurements and evaluation}

Each selected molecule is examined systematically in a series of measurements. For each buffer gas, the candidate gas is filled into the vacuum chamber with different mixing ratios. For each gas mixture, the experiment is performed at different settings of the experiment parameters gas number density $N$, electrode separation $d$, and applied DC voltage $U$. Displacement current waveforms are sampled, and typically a certain value $(E / N)$ is implemented for several different combinations of $(N, d, U)$. From these current curves, the swarm parameters electron drift velocity $w$, effective (ionization) rate constant $\nu_{\text {eff }} / N$, and diffusion time constant $N \tau_{D}$ are derived by regression methods [19]. Example results of $\nu_{\text {eff }} / N$ are shown in Figs. 5 and 6 for $\mathrm{C}_{3} \mathrm{~F}_{8}$ (Octofluoropropane) and $\mathrm{SF}_{6}$ mixed into Ar with small concentrations. The effect of increasing attaching gas concentrations can be judged in two ways: i) the increasing value of $\mathrm{E}_{\text {crit }}$ (for $\nu_{\text {eff }}=0$ ) and ii) the decreasing value of $\nu_{\text {eff }} / N$ for constant $E / N$ indicating an increased attachment rate. The resulting $\mathrm{E}_{\text {crit }}$ is listed in Table 3 for low percentage mixtures of both gases with $\mathrm{Ar}$ and $\mathrm{N}_{2}$. From these results, the effect of increasing concentration can be seen and the resulting electric strength of mixtures with different buffer gases in homogeneous fields can be compared.

\subsection{Space-Charge Density Effect}

Discharges in the two regimes, without and with influence of the space charge density, are typically called swarm and streamer, respectively $[21,48]$. In the context of this contribution, there are two reasons why the effects of space charge density need to be investigated.

Firstly, electron diffusion influences the development of discharges, such as the avalanche to streamer transition [48]. Generally, the electron density increases with increasing gas density. For swarms in $\mathrm{N}_{2}$, anisotropic diffusion was found with longitudinal diffusion coefficients $D_{\mathrm{L}}$ being smaller than the transversal $D_{\mathrm{T}}$ values $[49,50]$. For streamer heads, it was found $D_{\mathrm{L}}>D_{\mathrm{T}}[51]$. 


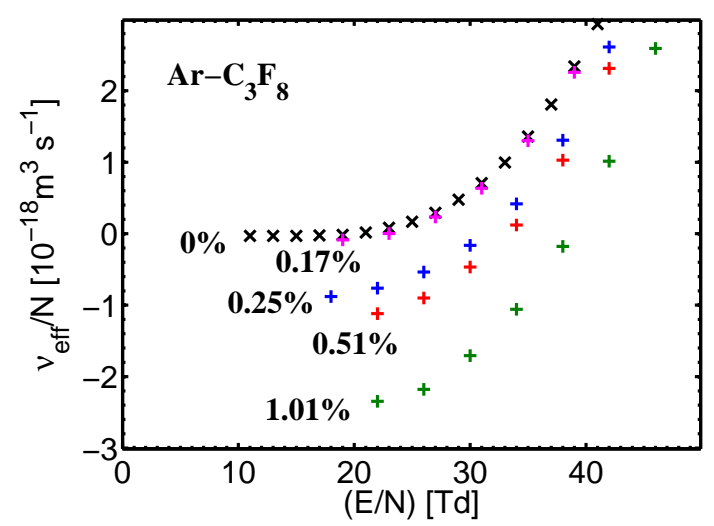

Fig. 5 Measurement of the effective ionization rate for low percentage mixtures of $\mathrm{C}_{3} \mathrm{~F}_{8}$ in Argon.

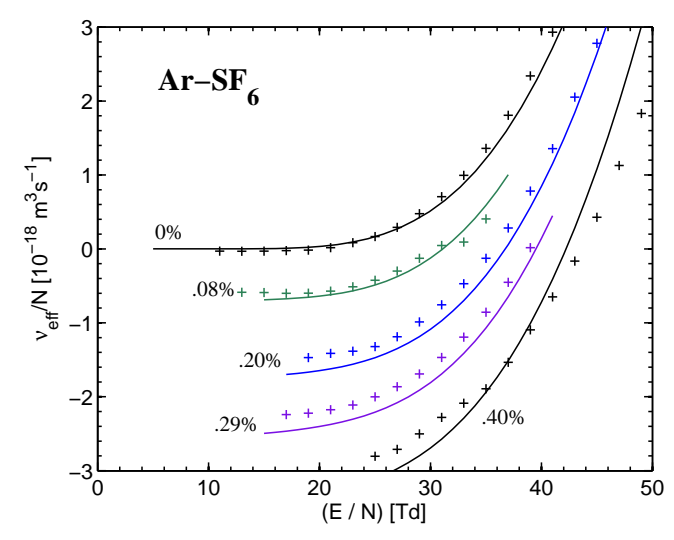

Fig. 6 Measured (+) and calculated with a two-term expanded Boltzmann equation [47] (dotted lines) effective ionization rate for low percentage mixtures of $\mathrm{SF}_{6}$ in Argon [19].

Table 3 Investigated mixing ratios of attaching gases in $\mathrm{Ar}$ and $\mathrm{N}_{2}$ and $E / N_{\text {crit }}$ [19].

\begin{tabular}{l|cc|cc}
\hline & \multicolumn{2}{|c|}{$\mathrm{SF}_{6}$} & \multicolumn{2}{c}{$\mathrm{C}_{3} \mathrm{~F}_{8}$} \\
\hline buffer & mixing & $(E / N)_{\text {crit }}$ & mixing & $(E / N)_{\text {crit }}$ \\
gas & ratio [\%] & {$[\mathrm{Td}]$} & ratio [\%] & {$[\mathrm{Td}]$} \\
\hline $\mathrm{Ar}$ & 0.08 & 30.5 & 0.17 & 25.3 \\
& 0.20 & 37.5 & 0.25 & 32.7 \\
& 0.29 & 41.0 & 0.51 & 34.2 \\
& 0.40 & 43.5 & 1.01 & 40.2 \\
\hline $\mathrm{N}_{2}$ & 0.25 & 134 & 0.37 & 94 \\
& 0.62 & 151 & 0.80 & 107 \\
& 1.01 & 161 & 1.50 & 118 \\
\hline
\end{tabular}

Secondly, to obtain large displacement current signals, it would be advantageous to study drifting swarms with a large number of electrons, i.e. to release the maximum number of electrons possible from the photocathode. However, it is important that the interaction of the drifting charge carriers with the background gas can be considered as independent of the swarm itself. That means the electric field generated by the space charge density of charge carriers should be negligible compared to the background field.

We contribute to these questions with measurements of longitudinal diffusion as a function of swarm electron number $n_{0}$ and gas density $N$ up to the regime where space charges strongly influence the electron swarm. We control the initial electron number $n_{0}$ by variation of the laser pulse energy incident on the photocathode. A variation of $n_{0}$ was feasible between 0.2 and $3.2 \times 10^{8}$.

Measurements have been made for 2,3 and $6 \mathrm{kPa}$ gas pressure at room temperature 290 to $295 \mathrm{~K}$. The accurate $\mathrm{N}_{2}$ density was $4.39,7.00$ and $14.5 \times 10^{23} \mathrm{~m}^{-3}$, respectively. The electrode distance $d$ was set between 11 to $18 \mathrm{~mm}$ in steps of $1 \mathrm{~mm}$, and $(E / N)$ was equivalent to 108,110 and $112 \mathrm{Td}$.

Three sets of measured diffusion time constants $\tau_{D}$ are shown in Fig. 7. It is apparent that larger $n_{0}$ resulted in higher $\tau_{\mathrm{D}}$-values. The data sets $n_{01}$ and $n_{02}$ clearly make up straight lines, where the data points almost coincide for the three $(E / N)$-values. The slope of these lines corresponds to the density normalized diffusion time constant $N \tau_{\mathrm{D}}$ [19]. Both data sets $n_{01}$ and $n_{02}$ yielded almost the same $N \tau_{\mathrm{D}}=4.35 \times 10^{14} \mathrm{sm}^{-3}$. The ordinate intersect of the regression lines corresponds to the initial width of the electron swarm. One can note from Fig. 7 that larger $n_{0}$ produce a larger initial width. The data set $n_{03}$ of Fig. 7 shows two effects. A nonlinear relation between $1 / d$ and $\tau_{\mathrm{D}} / d$ appeared due to extraordinarily large diffusion constants for the longer drift distances. Secondly, three distinct $\tau_{\mathrm{D}}$-values are discernible for every one setting of $d$, because $n_{0}$ was decreasing during recording of 
the data set due to rapid deterioration of the photocathode quantum efficiency. It was not possible to derive $N \tau_{\mathrm{D}}$ from the latter data set, but it gives a clear indication of the strong influence of swarm electron numbers on $\tau_{\mathrm{D}}$.

Accordingly we determined $N \tau_{\mathrm{D}}$ for the remaining data sets. All the results have been interpolated to $110 \mathrm{Td}$. The data for 2 and $6 \mathrm{kPa}$ are presented in 8 , where the $N \tau_{\mathrm{D}}$-values are much higher for $6 \mathrm{kPa}$ than for $2 \mathrm{kPa}$. However, there seems to be no clear dependence between $n_{0}$ and $N \tau_{\mathrm{D}}$, and it is justified to deduce one $N \tau_{\mathrm{D}^{-}}$ value for every $N$ setting. We chose the median, as indicated by horizontal dotted lines in Fig. 8 .

Fig. 9 presents the median $N \tau_{\mathrm{D}}$ for the three $N$ settings. Using a Monte Carlo method [50] the electron flux diffusion was simulated. The simulation reproduced the $E / N$ conditions of the measurements, but assumed no electron-electron interactions. The simulated longitudinal and transversal diffusion time constants $N \tau_{\mathrm{L}}=$ $2 N D_{\mathrm{L}} / w^{2}=2.48 \times 10^{14} \mathrm{sm}^{-3}$ and $N \tau_{\mathrm{T}}=2 N D_{\mathrm{T}} / w^{2}=4.33 \times 10^{14} \mathrm{sm}^{-3}$ were plotted as dashed horizontal lines in Fig. 9. The experimental $N \tau_{\mathrm{D}}$ are always higher than the simulated $N \tau_{\mathrm{L}}$, and for $6 \mathrm{kPa}$ the $N \tau_{\mathrm{D}}$ is even higher than $N \tau_{\mathrm{T}}$. It is apparent that electron flux diffusion is disturbed under the conditions of high electron densities.

Previously we have described a normalization technique for PT measurements, which is based on regression analyses of waveform parameters obtained for different $N$ settings [19]. The same method was applied to the present waveform data, and we have obtained density normalized electron swarm parameters as a function of $n_{0}$. For small $n_{0}$ the evaluation yielded $N \tau_{\mathrm{D}}=(2.61 \pm 0.05) \times 10^{14} \mathrm{sm}^{-3}$, a swarm drift velocity $w=$

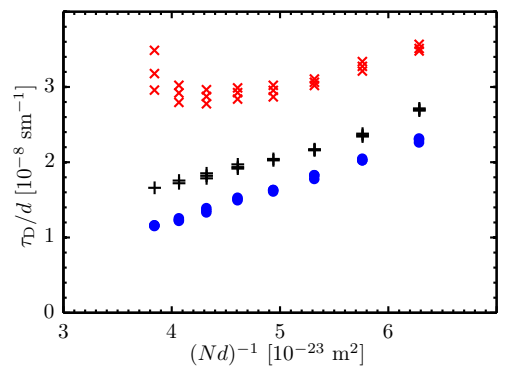

Fig. 7 Diffusion time constants $\tau_{\mathrm{D}}$ measured in $6 \mathrm{kPa} \mathrm{N} 2$ with initial electron number $n_{01}=3 \cdot 10^{7}(\bullet), n_{02}=10^{8}$ $(+)$ and $n_{03}=2$ to $3 \cdot 10^{8}(\times)$.

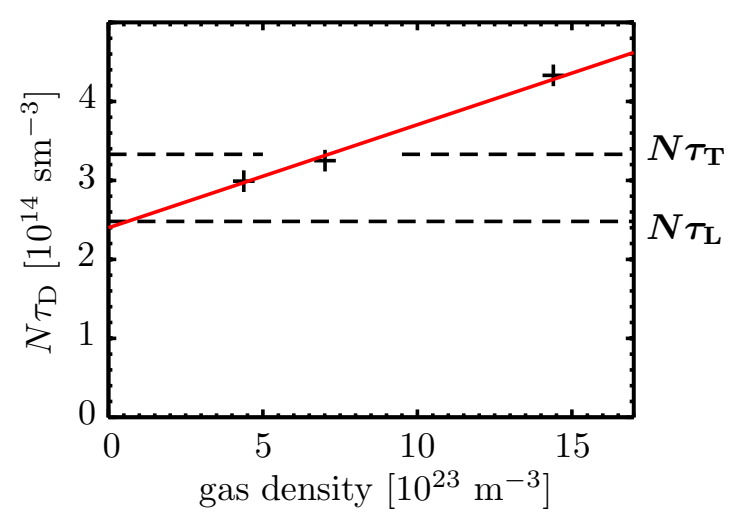

Fig. 9 Present median diffusion time constants $(+)$ with their linear trend (red solid lines), and the anisotropic flux diffusion coefficients (dashed) from a Monte Carlo method [50]. All the values are for $110 \mathrm{Td}$.

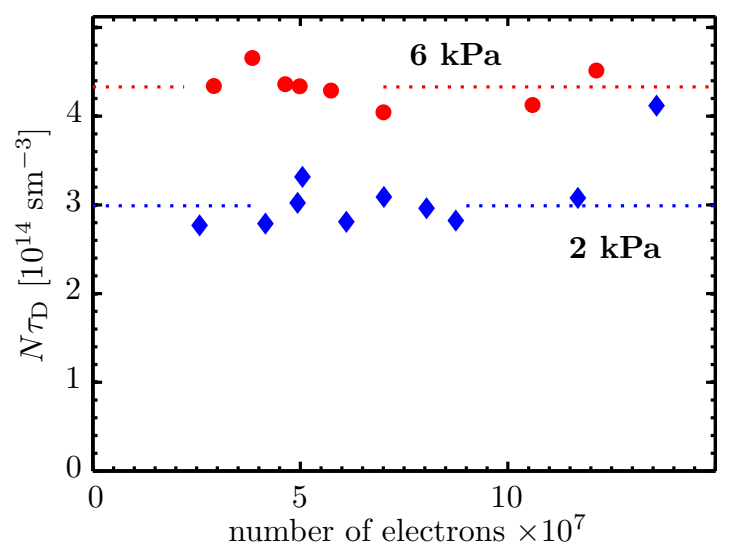

Fig. 8 Density normalized diffusion time constants measured at $2 \mathrm{kPa}(\bullet)$ or $6 \mathrm{kPa}(\bullet)$. The horizontal dotted lines represent their median values.

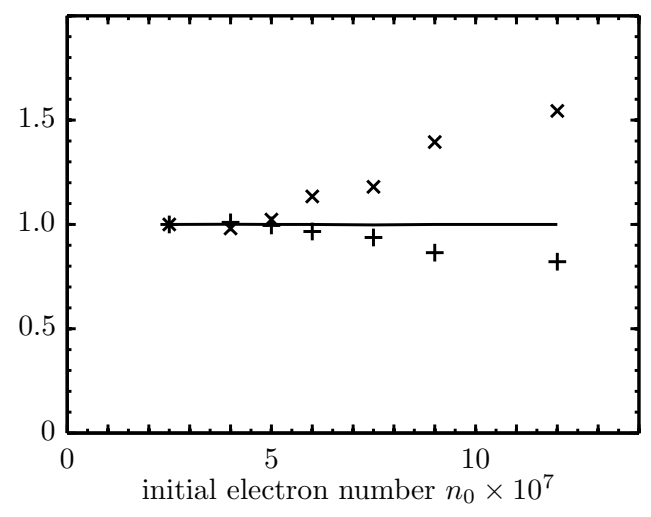

Fig. 10 Relation between initial swarm electron number $n_{0}$ and the swarm parameters $w$ (solid line), $\nu_{\text {eff }} / N(+)$ and $N \tau_{\mathrm{D}}(\times)$ for $110 \mathrm{Td}$ scaled to their values at $n_{0}=2.5 \times 10^{7}$. 
$1.176 \times 10^{5} \mathrm{~ms}^{-1}$, and an effective ionization rate $\nu_{\mathrm{eff}} / N=(2.07 \pm 0.03) \times 10^{-18} \mathrm{~m}^{3} \mathrm{~s}^{-1}$. Fig. 10 presents the results scaled to the values obtained for small $n_{0}$. The regression technique yielded almost the same $w$ for the whole range of $n_{0}$-values. However for $n_{0}>5 \cdot 10^{7}, N \tau_{\mathrm{D}}$ clearly increases with $n_{0}$, and $\nu_{\text {eff }} / N$ seems to decrease with $n_{0}$. From our methods we cannot conclude definitely if the observed decrease of $\nu_{\text {eff }} / N$ stems from the effect of space charges; it cannot be ruled out completely that it may stem from artifacts in the evaluation.

It is interesting to compare the dependence between $N \tau_{\mathrm{D}}$ and $n_{0}$ in figures 9 and 10. A scatter of the ordinates around the median $N \tau_{\mathrm{D}}$ is translated to a monotonic dependence. To our experience, the regression method reduces disturbing influences of the setup and improves the sensitivity of the PT method to reproduce the electron swarm evolution. Indeed, the regression result for small $n_{0}$ is only $5 \%$ higher than $N \tau_{\mathrm{L}}$.

The experimental data shown in Fig. 9 seem to indicate a linear relation between $N$ and $N \tau_{\mathrm{D}}$. If the linear trend is valid also for small $N$-values, the $N \tau_{\mathrm{D}}$-trend could be described with the linear parameter $\delta$ :

$$
N \tau_{\mathrm{D}}=N \tau_{\mathrm{L}}+N \delta
$$

From present data we found $\delta=1.3 \times 10^{-10} \mathrm{~s}$, and an ordinate intersect of $2.4 \times 10^{14} \mathrm{sm}^{-3}$, which is the extrapolation of measured $N \tau_{\mathrm{D}}$ to zero $N$. The extrapolation almost coincides with the simulated $N \tau_{\mathrm{L}}$.

To conclude this section, the presented swarm measurements demonstrated a strong increase of longitudinal diffusion coefficients with gas density and with the number of swarm electrons. Altogether that means an increase of diffusion coefficients with electron density, and thus we observed the dispersive force of space charges on electron swarms under the conditions of the present study. We emphasize the implications for fluid models, which are very commonly applied to represent the pre-breakdown phase in the atmosphere and in high voltage equipment: The proper diffusion type needs to be identified, and the transition between two diffusion types should be treated properly.

In addition, also the evaluation of the other swarm parameters is influenced. The limiting experimental conditions for direct determination of the longitudinal diffusion coefficients can be expressed as the product of gas pressure times swarm drift current, and our results indicate a limiting value of $0.02 \mathrm{PaA}$ in nitrogen. This limit must be respected during the swarm parameter measurements and is ensured by appropriate control algorithms of the experiment.

\section{Breakdown voltage prediction}

The swarm parameters are characteristic for a certain gas mixture and enable a quantitative comparison and evaluation. The critical electric field strength, for example, allows a direct comparison of the breakdown strength in homogeneous and slowly varying electric fields. To judge the applicability of a gas in GIS, however, this information is not sufficient. For this, one must be able to predict the breakdown voltage for all design parameters, e.g. different degrees of homogeneity (geometries), filling pressures, surface roughnesses, voltage wave shapes, and for all sort of potential defects and protrusions. To date, the breakdown processes in air and pressurized $\mathrm{SF}_{6}$ have been investigated intensely, but formulae for the breakdown voltage have mainly been derived for the most critical cases. These are the positive leader breakdown in air for large gaps for AC or switching impulse voltage [52], and in $\mathrm{SF}_{6}$ the positive lightning impulse voltage for strongly inhomogeneous field distributions as they occur in GIS with long protrusions $[53,54,55]$. In defect free $\mathrm{SF}_{6}$ GIS, the breakdown mechanism depends on the applied voltage wave shape, polarity and surface roughness and it is not generally possible to state the most critical case. Recently, a complete comparison of the parameter dependencies of the breakdown mechanism and voltage in $\mathrm{SF}_{6}$ has been made for all technical relevant situations [56]. For this, the first electron occurrence, the streamer inception field and the leader propagation have been estimated and for each condition the most critical was selected. By this, it was possible to estimate the breakdown voltage for all technical relevant conditions (geometries) in $\mathrm{SF}_{6}$ insulated equipment for all relevant voltage wave shapes. The model is physically motivated from the observation that the dominant breakdown process in strongly electro-negative gases occurs via the stepped leader mechanism [57]. The model uses only a few parameters that are derived indirectly via measurements or are guessed. It can thus be assumed that this model can be transferred to other electro-negative gases and only a few parameters need to be remeasured. If this assumption holds true, the breakdown voltages in gas insulated equipment with new insulation gas mixtures can be predicted and evaluated without performing extensive breakdown experiments. 
The above mentioned leader propagation model in $\mathrm{SF}_{6}$ was presented in [58, 59], experimentally validated for different cases in $[60,61]$. Experiments to test this model in other electronegative gases are currently in preparation.

\section{Summary and Conclusion}

We have presented a new efficient procedure to identify and quantify candidate molecules for insulating gas mixtures as replacement for $\mathrm{SF}_{6}$ in high-voltage gas insulated equipment. It consists of three steps: an efficient pre-selection based on numerical calculations of molecular properties, the measurement of swarm parameters of these gases in mixtures with buffer gases and the calculation of breakdown voltages for technical relevant conditions using these swarm parameters. Whereas the first two steps are successfully implemented and tested already, the last step is not yet fully proven to work reliably for all candidate electronegative gases and gas mixtures.

Even though this procedure is very powerful and candidate gases are efficiently characterized, numerous additional questions need to be raised and answered before an $\mathrm{SF}_{6}$ replacement gas is widely used and accepted. The long-term stability of the gas molecule and the gas mixtures has to be investigated, the dissociation products have to be examined for their environmental impact (in particular in combination with humidity), and it needs to be researched if the GIS design must be adapted.

\section{References}

[1] T. Rokunohe, Y. Yagihashi, F. Endo, and T. Oomori, Fundamental Insulation Characteristics of Air, $\mathrm{N}_{2}, \mathrm{CO}_{2}, \mathrm{~N}_{2} / \mathrm{O}_{2}$, and $\mathrm{SF}_{6} / \mathrm{N}_{2}$ Mixed Gases, Electrical Engineering in Japan, 155(3), 9-17 (2006).

[2] W. Boeck et al. (Task Force D1.03.10), $\mathrm{N}_{2} / \mathrm{SF}_{6}$ Mixtures for Gas Insulated Systems, Cigre Technical Brochure 260 (2004).

[3] G. Biasiutti, W. Zaengl, New dielectric gases for high-voltage engineering-investigations in a homogeneous field (in German), Bulletin ASE/UCS 74(3), 140-147 (1983).

[4] K. P. Brand, Dielectric strength, boiling point and toxicity of gases - different aspects of the same basic molecular properties, IEEE Trans. Dielectr. Electr. Insul. 17, 451-456 (1982).

[5] J. C. Devins, Replacement Gases for $\mathrm{SF}_{6}$, IEEE Trans. Dielectr. Electr. Insul. 15(2), 81-86 (1980).

[6] L. G. Christophorou, J. K. Olthoff, and D. S. Green, Gases for Electrical Insulation and Arc Interruption: Possible Present and Future Alternatives to Pure $\mathrm{SF}_{6}$, National Inst. Sci. Techn. (NIST), Washington DC, USA, Technical Note 1425 (1997).

[7] M. Rabie, D. A. Dahl, S. M. A. Donald, M. Reiher, and C. M. Franck, IEEE Trans. Dielectr. Electr. Insul., accepted for publication (2013).

[8] Th. Aschwanden and G. Biasiutti, Dielectric strength of hexafluoropropylene $\left(\mathrm{C}_{3} \mathrm{~F}_{6}\right)$, J. Phys. D: Appl. Phys. 14, L189$92(1981)$

[9] H. Kojima, Breakdown Characteristics of $\mathrm{N}_{2} \mathrm{O}$ Gas Mixtures for Quasiuniform Electric Field under Lightning Impulse Voltage, IEEE Trans. Dielectr. Electr. Insul. 14(6), 1492-1497 (2007).

[10] M. Hikita, S. Ohtsuka, S: Okabe, and S. Kaneko, Insulation characteristics of gas mixtures including perfluorocarbon gas, IEEE Trans. Dielectr. Electr. Insul. 15(4), 1015-1022 (2008).

[11] H. Katagiri, H. Kasuya, H. Mizoguchi, and S. Yanabu, Investigation of the Performance of $\mathrm{CF}_{3} \mathrm{I}$ Gas as a Possible Substitute for $\mathrm{SF}_{6}$, IEEE Trans. Dielectr. Electr. Insul. 15(5), 1424-1429 (2008).

[12] W. Hauschild and W. Mosch, Statistical Techniques for High-Voltage Engineering, Institution Of Engineering And Technology (1992)

[13] P. Simka, U. Straumann, C. M. Franck, $\mathrm{SF}_{6}$ High Voltage Circuit Breaker Contact Systems Under Lightning Impulse and Very Fast Transient Voltage Stress, IEEE Trans. Dielectr. Electr. Insul. 19(3), 855-864 (2012).

[14] M. Koch, U. Straumann, C. M. Franck, Determination of Waiting Times between Successive Breakdown Experiments, Conference on Electrical Insulation and Dielectric Phenomena (CEIDP), Montreal, Canada, 349-352 (2012).

[15] M. Braun, S. Marienfeld, M. W. Ruf, H. Hotop, High-resolution electron attachment to the molecules $\mathrm{CCl}_{4}$ and $\mathrm{SF}_{6}$ over extended energy ranges with the (EX)LPA method, J. Phys. B - Atomic, Molecular and Optical Physics, 42(12) AR $125202(2009)$.

[16] M. Allan, Measurement of absolute differential cross sections for vibrational excitation of $\mathrm{O}_{2}$ by electron impact, J. Phys. B: At. Mol. Opt. Phys. 28, 5163 (1995).

[17] M. Vinodkumara, K. Korotb, and P. C. Vinodkumar, Computation of the electron impact total ionization cross sections of $\mathrm{C}_{n} \mathrm{H}_{(2 n+1)} \mathrm{OH}$ molecules from the threshold to $2 \mathrm{keV}$ energy range, International Journal of Mass Spectrometry, 305(1), 2629 (2011). 
[18] S. J. Buckman and M. J. Brunger, A Critical Comparison of Electron Scattering Cross Sections measured by Single Collision and Swarm Techniques, Aust. J. Phys. 50(3), 483-509 (1997).

[19] D. A. Dahl, T. H. Teich, and C. M. Franck, Obtaining precise electron swarm parameters from a pulsed Townsend setup, J. Phys. D: Appl. Phys. 45, 485201 (2012).

[20] K. Kumar, The physics of swarms and some basic questions of kinetic theory, Physics Reports 112(5), 319375 (1984).

[21] H. Raether, Electron Avalanches and Breakdown in Gases, Butterworths, London (1964).

[22] NIST/SEMATECH, e-Handbook of Statistical Methods, http://www.itl.nist.gov/div898/handbook/ (2012).

[23] N. R. Draper and H. Smith, Applied Regression Analysis, Wiley Series in Probability and Statistics (1998).

[24] R. Ahlrichs, M. Bär, M. Häser, H. Horn, and C. Kölmel, Electronic structure calculations on workstation computers: The program system turbomole, Chemical Phys. Lett. 162, 165-169 (1989).

[25] G. Biasutti, Neue Isoliergase für gekapselte Hochspannungsanlagen (in German), Ph.D. Thesis, Diss. ETH Nr. 7683 (1985).

[26] National Inst. Sci. Techn. (NIST), USA, Chemistry WebBook, http://webbook.nist.gov/chemistry/ (2011).

[27] R. E. Wooton, M. R. Kegelman, et al., Gases Superiour to $\mathrm{SF}_{6}$ for Insulation and Interruption, Electric Power Research Insititute (EPRI), EL-2620 (1982).

[28] A. Kazakov, M. O. McLinden, and M. Frenkel, Computational Design of New Refrigerant Fluids Based on Environmental, Safety, and Thermodynamic Characteristics, Ind. Eng. Chem. Res. 51(38), 1253712548 (2012).

[29] W. Zaengl, Electronegative gases: present state of knowledge and applications, future prospects, Nordic Insulation Symposium (Nord-IS 88), I/1-39 (1988).

[30] L. G. Christophorou, Nuclear Instruments and Methods in Physics Research A268, 424-433 (1988).

[31] L. G. Christophorou, and J. K. Olthoff, Electron interactions with $\mathrm{SF}_{6}$, J. of Phys. and Chem. Reference Data, 29(3), 267-330 (2000).

[32] G. Raju, Chapter 116: Perfluoropropane (C3F8) in Gaseous Electronics Tables, Atoms, and Molecules, CRC Press (2011).

[33] X. Jiang, C. N. Berglund, A. E. Bell, W. A. Mackie, Photoemission from gold thin films for application in multiphotocathode arrays for electron beam lithography, J. Vac. Sci. Technol. B 16, 3374 (1998).

[34] A. Janzen et al., A pulsed electron gun for ultrafast electron diffraction at surfaces, Rev. Sci. Instr. 78, 13906 (2007).

[35] R. Karrer et al., Design of a miniature picosecond low-energy electron gun for time-resolved scattering experiments, Rev. Sci. Instr. 72, 4404 (2001).

[36] Y. Wang, L. G. Christophorou, and J. K. Verbrugge, Effect of temperature on electron attachment to and negative ion states of $\mathrm{CCl}_{2} \mathrm{~F}_{2}$, J. Chem. Phys. 109, 8304 (1998).

[37] J. Kopyra, J. Wnorowska, M. Forys, and I. Szamrej, A new apparatus for measuring rate constants and activation energies of thermal electron capture processes in the gas phase, International Journal of Mass Spectrometry 268, 6065 (2007).

[38] J. de Urquijo, $\mathrm{Is}_{\mathrm{CF}} \mathrm{I}$ a good gaseous dielectric? A comparative swarm study of $\mathrm{CF}_{3} \mathrm{I}$ and $\mathrm{SF}_{6}$, J. Phys.: Conf. Ser. 86, 012008 (2007).

[39] H. F. A. Verhaart and P. C. T. van der Laan, The influence of water vapor on avalanches in air, J. Appl. Phys. 55(9), 3286-3292 (1984).

[40] R. J. Wrobel and S. Becker, Carbon and sulphur on $\operatorname{Pd}(111)$ and $\operatorname{Pt}(111)$ : Experimental problems during cleaning the substrates and impact of sulphur on the redox properties of $\mathrm{CeO}_{x}$ in the $\mathrm{CeO}_{x} / \mathrm{Pd}(111)$ system, Vacuum 84, 1258 (2010).

[41] T. Aschwanden, Ph.D. dissertation ETH Zurich, Diss. ETH Nr.7931 (1985).

[42] D. A. Price, J. Lucas, J. L. Moruzzi, Ionization in oxygen-hydrogen mixtures, J. Phys. D: Appl. Phys. 5, 1249 (1972).

[43] M. Yamaji, Y. Nakamura, Y. Morokuma, Measurements of ionization and attachment coefficients in $0.468 \%$ and $4.910 \%$ c- $\mathrm{C}_{4} \mathrm{~F}_{8} / \mathrm{Ar}$ mixtures and pure c- $\mathrm{C}_{4} \mathrm{~F}_{8}$, J. Phys. D: Appl. Phys. 37, 432 (2004).

[44] D. Stelman, J. L. Moruzzi, A. V. Phelps, Low energy electron attachment to ozone using swarm techniques, J. Chem. Phys. 56, 4183 (1972).

[45] V. K. Lakdawala, J. L. Moruzzi, Measurements of attachment coefficients in $\mathrm{NF}_{3}$-nitrogen and $\mathrm{NF}_{3}$-rare gas mixtures using swarm techniques, J. Phys. D: Appl. Phys. 13, 377 (1980).

[46] R. H. Fowler, The analysis of photoelectric sensitivity curves for clean metals at various temperatures, Phys. Rev. 38, 45 (1931).

[47] G. J. M. Hagelaar, L. C. Pitchford, Solving the Boltzmann equation to obtain electron transport coefficients and rate coefficients for fluid models, Plasma Sources Sci. Technol. 14, 722 (2005).

[48] C. Montijn and Ute Ebert, Diffusion correction to the RaetherMeek criterion for the avalanche-to-streamer transition, J. Phys. D: Appl. Phys. 39, 29792992 (2006).

[49] B. M. Penetrante, J. N. Bardsley, L. C. Pitchford, Monte carlo and boltzmann calculations of the density gradient expanded energy distribution functions of electron swarms in gases, J. Phys. D: Appl. Phys. 18, 1087 (1985).

[50] S. F. Biagi, Monte Carlo simulation of electron drift and diffusion in counting gases under the influence of electric and magnetic fields, Nucl. Instr. and Meth. A 421, 234 (1999).

[51] O. Eichwald, H. Bensaad, O. Ducasse, M. Yousfi, Effects of numerical and physical anisotropic diffusion on branching phenomena of negative-streamer dynamics, J. Phys. D: Appl. Phys. 45, 385203 (2012).

[52] W. Mosch, E. Lemke, A model of the breakdown process in long air spark-gaps, ETZ A 95(5), 256-260 (1974). 
[53] N. Wiegart, Inhomogeneous field breakdown in GIS - The Prediction of Breakdown Probabilities and Voltages Part I: Overview of a theory for inhomogeneous field breakdown in $\mathrm{SF}_{6}$, IEEE Trans. Power Delivery 3(3), 923-930 (1988).

[54] N. Wiegart, Inhomogeneous field breakdown in GIS - The Prediction of Breakdown Probabilities and Voltages Part II: Ion density and statistical time-lag, IEEE Trans. Power Delivery 3(3), 931-938 (1988).

[55] N. Wiegart, Inhomogeneous field breakdown in GIS - The Prediction of Breakdown Probabilities and Voltages Part III: Discharge development in $\mathrm{SF}_{6}$ and computer model of breadkown, IEEE Trans. Power Delivery 3(3), 939-946 (1988).

[56] M. Bujotzek and M. Seeger, Parameter Dependence of Gaseous Insulation in $\mathrm{SF}_{6}$, IEEE Trans. Dielectr. Electr. Insul., accepted for publication (2013)

[57] I. Gallimberti, Breakdown mechanisms in electronegative gases, Gaseous Dielectrics V, Knoxville, 61-80 (1987).

[58] M. Seeger, L. Niemeyer, and M. Bujotzek, Partial discharges and breakdown at protrusions in uniform background fields in $\mathrm{SF}_{6}$, J. Phys. D: Appl. Phys. 41, 185204 (2008)

[59] M. Seeger, L. Niemeyer, and M. Bujotzek, Leader propagation in uniform background fields in SF 6 , J. Phys. D: Appl. Phys. 42, 185205 (2009).

[60] M. Bujotzek and M. Seeger, Partial discharges and breakdown in $\mathrm{SF}_{6}$ at submillimeter defects, $16^{\text {th }}$ Int. Symp. High Voltage Eng., Cape Town, South Africa, 656-661 (2009).

[61] M. Bujotzek and M. Seeger, Leader propagation in non-uniform background fields in $\mathrm{SF}_{6}$, XVIII Int. Conf. Gas Discharges and their Applications, Greifswald, Germany, 326-329 (2010). 